INPLASY

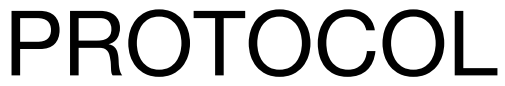

To cite: Zhou et al.

Effectiveness and Safety of

Electroacupuncture for

Depression: a systematic

review and meta-analysis.

Inplasy protocol 202210068.

doi:

10.37766/inplasy2022.1.0068

Received: 13 January 2022

Published: 14 January 2022

Corresponding author:

Zhuo Zhou

zhuozi0516@163.com

Author Affiliation:

Chengdu University of

Traditional Chinese Medicine

Support: National Natural

Science fund.

Review Stage at time of this submission: Data extraction Completed but not published.

Conflicts of interest:

None declared.

\section{Effectiveness and Safety of Electroacupuncture for Depression: a systematic review and meta-analysis}

Zhou, Z1; Xu, GX2; Huang, LY3; Tian, H4; Huang, FY5; Liu, YL6; Sun, $\mathrm{MS}^{7}$; Liang, $\mathrm{FR}^{8}$.

Review question / Objective: Is electroacupuncture a safe therapy for the treatment of depression? Is electroacupuncture effective for the treatment of depression, as compared with sham control, or conventional drugs?

Condition being studied: Depression is a mood disorder that causes sufferers to feel sadness, decreased interest, guilt, self-blame, loss of energy, and experience sleep disorders such as insomnia. People suffering from depression even feel they have no way out and have suicidal thoughts. In the United States, the prevalence of a major depressive disorder is $16.2 \% 1-3$. The 2010 Global Burden of Disease Study identified major depression as the second leading cause of disability worldwide and a leading cause of the burden of suicide and ischaemic heart disease. At present, depression patients are mainly treated with antidepressants, but the efficacy is extremely unstable. Studies have shown that acupuncture can help improve symptoms in patients with depression, but these clinical studies have not been systematically evaluated, and further confirmation is needed to confirm the efficacy of electroacupuncture in treating depression.

INPLASY registration number: This protocol was registered with the International Platform of Registered Systematic Review and Meta-Analysis Protocols (INPLASY) on 14 January 2022 and was last updated on 14 January 2022 (registration number INPLASY202210068).

\section{INTRODUCTION}

Review question / Objective: Is electroacupuncture a safe therapy for the treatment of depression? Is electroacupuncture effective for the treatment of depression, as compared with sham control, or conventional drugs?

Condition being studied: Depression is a mood disorder that causes sufferers to feel sadness, decreased interest, guilt, self- 
blame, loss of energy, and experience sleep disorders such as insomnia. People suffering from depression even feel they have no way out and have suicidal thoughts. In the United States, the prevalence of a major depressive disorder is $16.2 \% 1-3$. The 2010 Global Burden of Disease Study identified major depression as the second leading cause of disability worldwide and a leading cause of the burden of suicide and ischaemic heart disease. At present, depression patients are mainly treated with antidepressants, but the efficacy is extremely unstable. Studies have shown that acupuncture can help improve symptoms in patients with depression, but these clinical studies have not been systematically evaluated, and further confirmation is needed to confirm the efficacy of electroacupuncture in treating depression.

\section{METHODS}

Search strategy: The following eight databases will be searched from inception to 10th September 2021: PubMed, Embase, The Cochrane Library, Web of Science, China National Knowledge Infrastructure (CNKI), Chinese Biomedical Literature Database (CBM), VIP Database and WanFang Database. We followed key search terms and their potential combinations on PubMed: (1) clinical condition: depression, depressive disorders, depress, Journal Pre-proof "affective disorder, affective symptoms; (2) acupuncture terms: electroacupuncture, Electric acupuncture, EA, Electroacupuncture therapy, Electroacupuncture therapy, Electric acupuncture therapy; (3) study type: randomized controlled trial. We used "and" and "or" to connect the search terms.

Participant or population: Patients should suffer from depression.

Intervention: Electroacupuncture.

Comparator: Sham-acupuncture or drugs.

Study designs to be included: RCT.
Eligibility criteria: This study included all RCTs that have published Chinese or English articles about electroacupuncture for depression.All patients included in this study were diagnosed with depression according to Guidelines or other standards.

Information sources: The following seven databases will be searched from inception to 10th September 2021: PubMed, Embase, The Cochrane Library, Web of Science, China National Knowledge Infrastructure (CNKI), Chinese Biomedical Literature Database (CBM), VIP Database and WanFang Database.

Main outcome(s): The Hamilton depression scale (HAMD)

Additional outcome(s): Self-rating depression scale (SDS) and adverse reactions.

Data management: In this study, two reviewers ( $Z Z$ and $X G X$ ) searched the above databases respectively in no language limit. Two reviewers ( $Z Z$ and XGX) independently extracted data from the included trials. These data are recorded by Office 2019 . If the information is incomplete, we will contact the authors by phone or E-mail to acquire information. When disagreements arise in the research, they are resolved through discussion or consultation with a third reviewer (LFR) until consensus is reached.

Quality assessment / Risk of bias analysis: Two reviewers (HLY and TH) independently assessed the risk of bias in the final included studies using the risk of bias assessment tool by the Cochrane Collaboration. Two independent researchers (HFY and LYL) evaluated the legal quality by the Jadad scale.Any ambiguity in the evaluation process was resolved by LFR.

Strategy of data synthesis: Before synthesizing the data, we will unify the units of each outcome from different trials, depending on the International System of Units. And then, clinical data will be 
imported into STATA 15.0 to perform data synthesis. The risk ratio (RR)/odds ratio (OR) with a $95 \%$ confidence intervals (Cls) will be presented the results of dichotomous data analysis, while continuous outcomes will be investigated by using the mean difference (MD)/ the standardized mean difference (SMD) with 95\% Cls. Data will be synthesized with randomized effect model when the $1275 \%$ coming from the heterogeneity test. If there is considerable heterogeneity $(\mathbf{1 2 7 5 \%})$ in the trials, meta-analysis will not be performed. Random-effects models were used to analyze dichotomous and continuous data for risk ratio (RR) and standard mean difference (SMD) and 95\% confidence intervals $(95 \% \mathrm{Cl})$. $Q$ statistics were used to investigate the heterogeneity between studies, $P$ values $<0.10$ was considered an indicator of significant heterogeneity. When heterogeneity test $\mathbf{I} 275 \%$ or $\mathrm{P}<0.10$ ), each trial was removed from the population analysis. At the same time, sensitivity analysis was performed to identify studies that significantly affected the population effect. When we included enough trials, the funnel plot was visually examined, and Egger or Begg tests were used to quantitatively evaluate publication bias. The subgroup analysis or meta-regression were not conducted due to the few heterogeneities.

Subgroup analysis: In this case, we will try to identify the source of heterogeneity from both clinical and methodological aspects and a narrative, the qualitative summary will be provided. If sufficient trials are included, we will explore the following potential sources of heterogeneity using STATA software with subgroup analyses or metaregression from the variations in the characteristics of the trial participants, acupuncture treatments, sample size, methodological, etc.

Sensitivity analysis: Sensitivity analysis will be used to check the stability of the primary decision made in the review process. Several decision nodes will be considered within the process of the systematic review, such as small sample size studies, methodological weaknesses and missing data. The results of the sensitivity analysis will be presented in summary tables. The risk of bias in the review process, as indicated by the results of the sensitivity analysis, will be discussed.

Language: No limits.

Country(ies) involved: China.

Keywords: Electroacupuncture; Depression; Meta-Analysis.

Contributions of each author:

Author 1 - Zhuo Zhou.

Author 2 - Guixing Xu.

Author 3 - Liuyang Huang.

Author 4 - Hao Tian.

Author 5 - Fengyuan Huang.

Author 6 - Yilin Liu.

Author 7 - Mingsheng Sun.

Author 8 - Fanrong Liang. 